\title{
Cost-effectiveness Analysis of Utilization of Community Health Workers in Promotion of Maternal Health Services in Butere District, Rural Western Kenya
}

\author{
Caroline Akinyi ${ }^{1, *}$, Jeremie Nzanzu ${ }^{2}$, Dan Kaseje ${ }^{3}$, Rose Olayo ${ }^{4}$ \\ ${ }^{1}$ Department of Measurements in Health and Development, Great Lakes University of Kisumu (GLUK), (40103) 6683, Kisumu, Kenya \\ ${ }^{2}$ Department of Information Technology, Great Lakes University of Kisumu, (40100) 2224, Kisumu, Kenya \\ ${ }^{3}$ Department of Community Health and Development, Great Lakes University of Kisumu, (40100) 2224, Kisumu, Kenya \\ ${ }^{4}$ Department of Health Promotion, Great Lakes University of Kisumu, (40100) 2224, Kisumu, Kenya \\ *Corresponding Author: akinyicaroline12@yahoo.com
}

Copyright (C) 2014 Horizon Research Publishing All rights reserved.

\begin{abstract}
Cost-effectiveness analysis is a type of economic evaluation used to determine the best use of money available for medical care for informed decision making. This study carried out a cost-effectiveness analysis of using Community Health Workers (CHWs) as an integral part of Community Health Strategy implementation to promote uptake of maternal health services in Butere District, rural western Kenya. The main objective was to determine incremental cost-effectiveness ratio of utilization of CHWs in promotion of maternal health services in Butere District (intervention site) in selected Community Health Units. The costs and consequences of promoting maternal health services at the community level with CHWs were identified, measured, valued while using a quasi experimental study design. Annual cost incurred in utilizing CHWs to promote maternal health services in Butere District in 2011 was US\$ 30,124, effectiveness of utilization of CHWs was 1,205 yearly DALY averted. The incremental cost-effectiveness ratio was US\$25 per yearly DALY averted.Utilization of CHWs to promote maternal health services is a cost effective strategy in Butere District. Community Health Strategy should be up scaled as it is a cost effective intervention to promote uptake of maternal health services especially in the rural population where health seeking behaviours are low.
\end{abstract}

Keywords Cost-effectiveness Analysis, Community Health Workers, Maternal Health Services

\section{Introduction}

Cost-effectiveness analysis (CEA) is a form of economic evaluation and primary tool for comparing the estimation of cost of a health intervention alongside a measure of outcome (typically health gain). An intervention can be understood to be any activity, using human, financial, and other inputs, that aims to improve health. Effectiveness is measured in terms of averted deaths or averted Disability Adjusted Life Years. $[1,2,3]$.

Estimates of cost-effectiveness for a particular health intervention, say in terms of cost per life saved, reducing the risk of a health problem, reducing the severity or duration of an illness or disability when compared with that of another, indicate where funds could be allocated to maximize health gain. Applied to the evaluation of community-based health programs it enables a decision-maker to choose between two or more modes of delivery for the same intervention (e.g., hospital- versus community-based care in order to identify which represents the most efficient, or "cost-effective", use of resources),[1,2].

The estimated cost-effectiveness of a new intervention is compared with the cost-effectiveness of an existing intervention or with a fixed price cut-off point representing the assumed social willingness to pay for an additional unit of health. The implicit assumption that the required additional resources would need to be transferred from another health intervention or from another sector is rarely discussed. Furthermore, cost-effective analysis can be used to inform decision makers in the health sector regarding their policies or used in a formulaic way to determine resource allocation, [4]. Allocative efficiency of health systems supports analytical efforts to study the cost-effectiveness of a broad range of interventions.

New metric measure of cost- effectiveness is disability-adjusted life year (DALY) which was introduced by the World Health Organization and the World Bank in 1993 and has been used since, with some variations, for two related purposes. One is to measure the "burden of disease," the extent to which premature deaths and disabilities cause a loss of health status compared to everyone's living to old age in good health. The other purpose is to compare the value of health interventions that have multiple or different health outcomes occurring at different ages. In cost-effectiveness 
analysis, the DALY represents the number of years of disability-free life that would be gained from a particular health intervention. In high-income countries, some analyses include quality-adjusted life years (QALYs), an alternative measure of how much a year of life is diminished if a person suffers health limitations. This measure can account for people suffering from more than one illness or disability and in varying degrees, $[2,3]$. QALYs are used minimally in the Disease Control Priorities Project that focuses on burden of disease in low- and middle-income countries.

The limited studies available suggest that utilization of Community Health Workers (CHWs) increases the coverage and equity of health service delivery compared other healthcare promotion and provision alternatives [5]. However most studies, while useful and necessary for decision-makers, lack key elements of CHW programmes that do not lend themselves to economic analysis: institutional factors such as altruism, volunteerism, community norms, reciprocity and duty and these tend not to be reflected well in estimates of cost effectiveness. Hence cost-effectiveness analyses are insensitive to a range of social benefits (including community mobilization), which often constitute the strength of CHW programmes.

More recently the lack of progress with the Millennium Development Goals (MDG) and primary health care in many poor countries has encouraged those in favour of comprehensive primary health care to question whether the failure to address community care and participation effectively within health programmes is a major reason for poor sustainability and ineffective scaling-up of selective interventions of proven efficacy. This leads to the following question, does delivery of health systems that rely solely on government health facilities be mandatorily expanded to include the full range of potential channels in a setting and strong community-based approaches?, [6]. The focus on process within maternal and child health programmes must change to include greater accountability for intervention coverage at population level. A crucial policy question is whether specific community participation interventions aimed at women and their families have a direct effect on maternal health? If so, how do these interventions work most effectively, and how can they be up scaled? It is on this basis that there was re-emergence of community based healthcare, a system which supports utilization of CHWs to bridge the interface between community and the health system in Kenya.

A review of randomized controlled trials (RCTs) on the effects of Lay Health Workers (LHWs)/ CHWs interventions in improving Maternal and Child Health and addressing key high burden diseases revealed that the use of LHWs in health programmes shows promising benefits, compared to usual care, in promoting immunization and breastfeeding uptake; in reducing mortality and morbidity from common childhood illnesses; and in improving TB treatment outcomes [7]. To increase the availability of health services with low cost and high quality, CHWs were trained to offer basic health care to all residents under five years old in a small county in Sao Paulo State, Brazil [8]. These services included diagnosis, early treatment and management of diarrhea, infectious respiratory diseases, growth monitoring and incentives to complete basic immunization. In a three year period, 410 children received weekly home visits from CHWs of which of infant mortality rate (IMR) reduced by 4.2 times and the hospitalization rate decreased six times. Oral re-hydration therapy (ORT) was administered in 90 percent of the diarrhea episodes and full immunization became practically universal. The prevalence of growth stunting reduced by 27.1 percent and program costs averaged $\$ 12.90$ per capita per year and $\$ 8.12$ per child per month. In summary, the program was able to increase the availability of health services with relatively low cost, and to drastically reduce infant morbidity and mortality.

A systematic review of randomized controlled trials (RCTs) on the effects of Lay Health Workers (LHWs) interventions in improving $\mathrm{MCH}$ and addressing key high burden diseases in Low and Middle Income Countries was conducted, [7]. They documented that use of LHWs in health programmes shows promising benefits, compared to usual care, in promoting immunization and breastfeeding uptake; in reducing mortality and morbidity from common childhood illnesses; and in improving TB treatment outcomes. Little evidence is available regarding the effectiveness of substituting LHWs for health professionals or the effectiveness of alternative training strategies for LHWs.

A number of studies have examined the cost-effectiveness of specific programmes. One of the first papers to evaluate the "value for money" of CHW programmes, [9]. The study reports on a project in two locations in Kenya's Western Province. CHWs were trained for 12 weeks and deployed as providers of basic health care and promoters of selected health, sanitation and nutrition practices. A cost-benefit analysis was performed using the willingness-to-pay approach to compare the costs and benefits of the project. The evaluation illustrated a large net present value and a benefit-cost ratio of between 9.36 and 9.85 , depending on the choice of discount rate. The author concluded that the results were "...strongly in favour of decentralization of primary health care on similar lines in the rest of the country" [9].

In making such comparisons, it is relevant to examine both costs and outcomes of switching between alternatives, i.e. the incremental cost and outcomes of $\mathrm{CHW}$ relative to a stated alternative, which is sometimes a "do nothing" option [9]. In terms of the incremental costs of CHW programs, a comparison with hospital-based care would need to highlight not only changes in resource use to the health sector but also those to patients and the wider community.

The challenges of using cost effective analysis are: - cost data can be extremely hard to find in developing countries, cost-effectiveness is only one criterion for judging whether an intervention has merit and in some cases it cannot stand on its own and equity is also a concern, because it can be more cost-effective to serve many people in large urban centers, where the cost per outcome is relatively low. 
Providing the same service in a poor, rural area-where fewer patients are seen or where staff and other inputs are harder to make available - might be less cost-effective but more worthy of public investment because it is more equitable, [2,3].

Gaps identified are the lack of sufficient data on actual annual costs incurred and cost-effectiveness of health interventions in promotion of maternal health services at community level especially in a rural setting (Butere District) where health seeking behaviours are poor as there is no clear documentation on this aspect. This study will clearly calculate these costs in Butere District, a rural setting in Kenya and also the cost-effectiveness of this intervention for future reference.

\subsection{Problem Statement}

Community Health Strategy was adopted in Kenya in 2006, as strategy which supports utilization of CHWs to promote maternal health services in Kenya. However, despite its implementation, there is still a declining trend in key maternal health indicators which demands an economic evaluation on the strategy to determine its economic viability and feasibility for evidence based decision making as a comparison of trends since the 2003 Kenya Demographic Health Survey (KDHS), the analysis shows a continuing decline in the proportion of women who attend focused antenatal care visits; from $52 \%$ in 2003 to $47 \%$ in 2008-09 and $56 \%$ of births still take place at home with no assistance of a skilled attendant. This scenario is even worse in rural areas compared to urban areas, [10], and hence maternal mortality levels still remains a perennial problem in Kenya and remains high at 488 deaths per 100,000 live births.

However this poses as a major challenge as calculation of cost and cost effectiveness of CHWs interventions in maternal health services are rarely mentioned in research studies concerning utilization of CHWs in medical care assessment. Dower et al. [11] illustrated the magnitude of lack of this information in the first careful look at CHW financing. The review found only six rigorous studies with economic and cost information, and these yielded insufficient data to evaluate the cost-effectiveness of CHW interventions relative to other community health interventions [12]. In the secondary literature, one sees repeated mentions of the same few studies that address costs. Moreover, since funding of CHWs programmes is usually scarce and it is therefore important to find the best value for money invested in this area.

The few studies available with evidence of costing or cost-effectiveness analysis, lack the element of generalizability due to the different contexts in which different health system setting exist. It is on this basis that this study calculated the incremental cost-effectiveness ratio of utilizing CHWs to promote maternal health services in Butere District, rural western Kenya.

\subsection{Research Objective}

To determine the incremental cost-effectiveness ratio of utilizing Community Health Workers to promote maternal health services to women of the reproductive age in Butere District (rural setting).

\subsection{Study Limitation}

A major limitation of this study is that it is entirely based on reports by health facilities and CHWs, hence there may be an aspect of miss-reporting and recall bias as well.

\section{Materials and Methods}

\subsection{Study Design}

This study used quasi experimental study design of selected Community Health Units in Butere District (intervention site) and Mumias Districts (non-intervention site) respectively.

The costs and consequences of promoting maternal health services at the community level using the CHWs was identified, measured, valued. This was a form of incremental cost-effectiveness analysis that considered costs and health consequences of promoting maternal health services from both the health system and societal perspective. The health consequences of the intervention were identified and measured in terms of coverage of the maternal health services with and without utilization of CHWs. The incremental cost-effectiveness ratio in terms of incremental cost per effect achieved for the interventions was calculated.

\subsection{Study Site}

The study was conducted in selected Community Health Units in Butere (Bubala, Mutoma, Shitari and Shibembe Community Health Units) and Mumias (Malaha, Emakhwale, Lusheya and Shianda Sub-locations) Districts respectively of Kakamega County, in Western Kenya. Butere District is located $430 \mathrm{~km}$ while Mumias District is located $445 \mathrm{~km}$ away from Nairobi which is the capital city of Kenya.

\subsection{Study Population}

This composed of all the mothers of reproductive aged 15-49 years who sought maternal health services (focused antenatal care and health facility delivery) at the facility in the year 2011, either on their own initiative or through referral by CHWs in the selected community health units (CHU).

\subsection{Sampling Design}

This study was based on multi-stage sampling design. The 
first stage was purposive sampling of the area because the selection of the site was based on the site being operated on by GLUK as partnership research site and there is implementation of Community Health Strategy (CHS) in Butere Sub-county (intervention site) and no operation of GLUK and no implementation of CHS in Mumias Sub-county (non intervention site). The study concentrated on the research sites which were the pioneer Community Unit (CU) established and used to implement the community health strategy and they are actively running up to date.

The second sampling stage was a complete enumeration of all the pregnant women aged 15-49 who received focused antenatal care and had health facility delivery in the year 2011 in the selected sites who meet the inclusion criteria of the study.

\subsection{Data Collection}

Training of research assistants and supervisors was undertaken. The training content focused on the rationale of the research, process, questionnaire, interviewing skills and research ethical issues. The questionnaires were pre-tested on a small group of clients for clarity, validity and reliability of the questions and appropriate adjustments made before commencement of the actual data collection.

For this study the District Health Management Team (DHMT) trainings and meetings are initially done to initiate the concept of the CHS and the Community Health Extension Worker (CHEW) training was meant to train both the Community Health Committee (CHC) and CHWs on household registration. Once this is done then the community health unit is said to be running.

Weekly meetings held were captured first on weekly basis, then interviews conducted weekly by identifying the key informant person who attended the meetings and were able to give details of the number of persons who attended, agenda discusses, time taken, materials used during the meetings and even the most common type of transport used by the attendants including details of allowances given if any in order to calculate the costs incurred.

The tools were in the following sets: a tool capturing mainly the meetings at the DHMT level in relation to the community health strategy, another tool capturing all trainings done by the DHMT level in relation to the community health strategy, a household registration tool which represented the data collected by the CHWs on mothers attending focused antenatal care and had health facility delivery while the last tool captured all the $\mathrm{CHC} / \mathrm{CHWs}$ meetings running in continuity of the community health strategy.

In all the tools the variables captured were costs of materials used during the trainings and meetings, the manpower present, any allowance given if it was, the most commonly used mode of transport, starting time and ending time which were all adding up to how much it costs to have a meeting or training in relation to the community health strategy, number of mothers who attended focused antenatal care and had health facility delivery as a result of community health strategy (intervention) and number of mothers who attended focused antenatal care and had health facility delivery at their own initiative (non intervention) . The data was collected over a period of one year, January to December 2011.The final figures were summarized to show cost-effectiveness of utilization of CHWs in the promotion of uptake of maternal health services.

\subsection{Data Processing and Analysis}

Filled in questionnaires were checked for completeness and consistency of responses. Data collected were cleaned, edited, coded and any errors corrected. Hard copies of all questionnaires were used to counter check information electronically entered.

Data were entered in on weekly basis by capturing all activities that had been done the previous week from the key informant person first which were then followed by interviewing the person on details like materials used in the tool. During data entry there were no merging of the tools and each tool was treated on its own.

Statistical Package for Social Sciences (SPSS version 16) and Microsoft excel were used for data entry, analysis and presentation. Descriptive statistics were used to find the mean, unit and total costs of implementing Community Health Strategy. Inferential statistics was used to estimate the effectiveness of the intervention (t-test statistic) and finally the incremental cost-effective ratio was calculated.

\section{Results}

\subsection{Annual Cost incurred in utilizing CHWs to Promote Maternal Health Services in Butere District in the Year 2011}

The cost of implementing Community Health Strategy while utilizing Community Health Worker to promote maternal health services for the whole year of 2011 was calculated as shown in Table 1 below

The highest cost incurred in was time (indirect cost). Time (CHC/CHWs/ Community) was calculated at a rate of Kshs.250 per day which is the amount earned for cultivating in a farm in a day (7a.m- $4.00 \mathrm{pm}=9$ hours) since major source of income of households in Butere District is farming. This rate was multiplied by the number of days which CHWs and community members spent in promoting or utilizing maternal health services through CHS. Moreover, there were a total of 28 meetings on CHS in the year 2011 and each lasted averagely for 3.5 hours. This is the amount CHWs and community members would have made instead of promoting or utilizing maternal health services (opportunity cost).

On the other hand, the time for CHEWs and CHWs meetings was calculated on the supervisory meetings and trainings held during the implementation of CHS in the promotion of uptake of maternal health services. 
Table 1. Annual costs incurred in running a CHU in utilizing CHWs to promote uptake of maternal health services in Butere District.

\begin{tabular}{|c|c|c|c|}
\hline Cost items & $\begin{array}{c}\text { CHEWs \& CHWs meetings } \\
\text { Kshs( USD) }\end{array}$ & $\begin{array}{c}\text { CHC/CHW meetings } \\
\text { Kshs(USD) }\end{array}$ & Total Kshs (USD) \\
\hline Writing pens & $3,240($ US\$ 39$)$ & $6120($ US\$74) & 9360(US\$113) \\
\hline Flip charts & $1,240($ US\$ 15$)$ & $865(\mathrm{US} \$ 10)$ & $2105(\mathrm{US} \$ 25)$ \\
\hline Felt pens & $4,000(\mathrm{US} \$ 48)$ & $8,500(\mathrm{US} \$ 102)$ & $12,500(\mathrm{US} \$ 150)$ \\
\hline Notebooks & $14,150($ US\$170) & $20,050($ US\$242) & $34,200($ US\$412) \\
\hline Foolscaps & 578(US\$7) & $1,000(\mathrm{US} \$ 12)$ & $1,578(\mathrm{US} \$ 19)$ \\
\hline Allowances & $1,250(\mathrm{US} \$ 15)$ & - & $1,250(\mathrm{US} \$ 15)$ \\
\hline Airtime & - & $2,500(\mathrm{US} \$ 30)$ & $2,500(\mathrm{US} \$ 30)$ \\
\hline Photocopies & $16,000(\mathrm{US} \$ 193)$ & 84(US\$1) & $16,084(\mathrm{US} \$ 194)$ \\
\hline Masking tapes & $600($ US\$7) & - & 600 (US\$7) \\
\hline Seats & $1,560($ US\$ 19$)$ & - & $1560(\mathrm{US} \$ 19)$ \\
\hline Time & $16,416($ US $\$ 198)$ & $527,000($ US\$6349) & $543,416($ US\$6547) \\
\hline Total & $59,034($ US\$711) & $566,119($ US\$6820) & $625,153($ US\$7531) \\
\hline \multicolumn{4}{|c|}{ Exchange Rate in 2011: 1 US Dollar $=83$ Kenyan Shillings } \\
\hline
\end{tabular}

Table 2. Annual costs incurred classification (type and perspective) in running a CHU in utilizing CHWs to promote uptake of maternal health services in Butere District.

\begin{tabular}{|c|c|c|c|}
\hline Type & Perspective & Cost(Kshs) & Cost( US\$) \\
\hline Direct costs & Health system & 42,618 & 513 \\
\hline Direct costs & Societal & 39,119 & 471 \\
\hline Indirect costs & Health system & 16,416 & 198 \\
\hline Indirect costs & Societal & 527,000 & 6349 \\
\hline Total & \multicolumn{4}{|c|}{ Exchange Rate in 2011: 1 US Dollar = 83 Kenyan Shillings } \\
\hline
\end{tabular}

The health system invested more in terms of direct costs (Human resource and allowances) while the society invested more in indirect costs (Time) spent in promoting maternal health services. The major investors in Community Health Strategy are the CHWs who are also the community members.

Four Community Health Units were purposively sampled on the basis of them being intervention sites in Butere District (Bubala, Mutoma, Shitari and Shibembe Community Units) hence the total cost given above in Table 3.0 is multiplied by four.

Annual cost incurred in utilizing Community Health Workers to promote uptake of maternal health services during implementation of Community Health Strategy in Butere District is given by:

$$
\begin{gathered}
\text { Kshs. } 4 \times 625,153=\text { Kshs. } 2,500,612 \\
\text { US\$ } 4 \times 7531=\text { US } \$ 30,124
\end{gathered}
$$

On the hand, the cost of implementing Community Health Strategy while using Community Health Workers to promote maternal health services in the non intervention site (Mumias District) was null. This is because since there was no intervention there was zero incremental cost incurred in its implementation.

\subsection{Effectiveness of Utilization of Community Health Workers to Promote Maternal Health Services (Focused Antenatal Care and Health Facility Delivery) in Butere District in 2011}

There was a statistically significant increase $(\mathrm{p}<0.05)$ in the proportion of women who had FANC (53\%-66\%) and also had Health Facility Delivery (35\%-48\%). However, more women went for FANC but the proportion reduces when it comes to Health Facility Delivery during childbirth even after intervention through Community Health Strategy implementation through utilization of CHWs to promote uptake of maternal health services in Butere District as shown in Figure 1.

On the hand, for the non intervention site (Mumias District) there was no significant difference in the proportion of women who Focused Antenatal Care and had Health Facility Delivery.

There was a statistically significance difference in means between Focused Antenatal Care attendance (FANC) and Health Facility Delivery in Butere District, 2011. The negative $\mathrm{t}$ - statistic indicated that while the proportion of women who had FANC was higher, the proportion of women who had Health Facility delivery was relatively lower as shown in Table 3, (i.e. there was an inverse relationship between the two indicators). On the other hand, there was no statistically significance mean difference between FANC attendance and health facility delivery in Mumias District $(\mathrm{p}>0.05)$. 


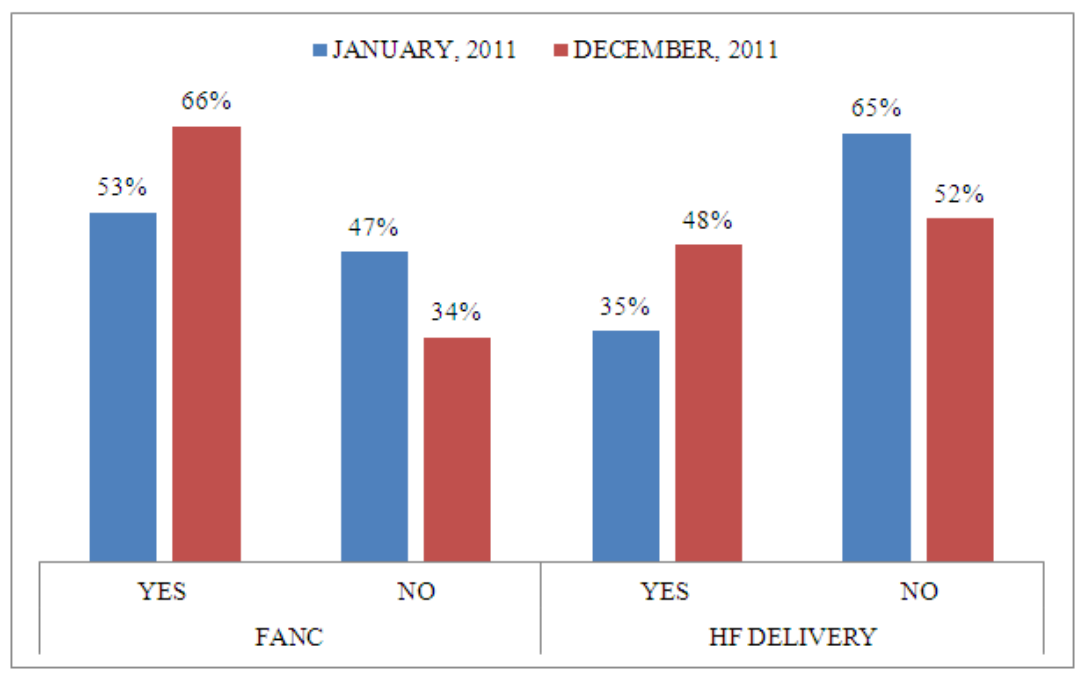

Figure 1. A Graph of FANC attendance and Health Facility Delivery in Butere District, January -December, 2011.

Table 3. T-test for difference in means of FANC attendance and Health Facility Delivery in Butere Sub-county, 2011

\begin{tabular}{|c|c|c|c|c|c|c|c|c|}
\hline \multirow{3}{*}{$\begin{array}{c}\text { Focused } \\
\text { Antenatal } \\
\text { Care and } \\
\text { Health } \\
\text { Facility } \\
\text { Delivery }\end{array}$} & \multirow[t]{2}{*}{ Mean } & \multirow[t]{2}{*}{$\begin{array}{l}\text { Standard } \\
\text { deviation }\end{array}$} & \multirow{2}{*}{$\begin{array}{c}\text { Standard } \\
\text { Error of } \\
\text { Mean }\end{array}$} & \multicolumn{2}{|c|}{$\begin{array}{l}\text { 95\% Confidence } \\
\text { Interval of the } \\
\text { Difference } \\
\end{array}$} & \multirow[t]{2}{*}{$\mathrm{T}$ statistic } & \multirow[t]{2}{*}{$\begin{array}{l}\text { Degrees of } \\
\text { freedom }\end{array}$} & \multirow[t]{2}{*}{$\mathrm{p}$-value } \\
\hline & & & & Lower & Upper & & & \\
\hline & -0.174 & 0.485 & 0.008 & -0.188 & -0.159 & -23.011 & 4136 & 0.001 \\
\hline
\end{tabular}

\subsection{Valuation of Health Effect}

Table 4. WHO framework for effects of intervention packages for sub-Saharan African countries with very high child and adult mortalities

\begin{tabular}{|c|c|c|}
\hline Intervention: Codes and Descriptions & Coverage & Yearly DALYs averted(million) \\
\hline MNH-1: Community Newborn Care Package & $50 \%$ & 10,752 \\
\hline MNH-2: Support for Breastfeeding Mothers & $50 \%$ & 9,118 \\
\hline MNH-7:Normal Delivery by a Skilled Attendant & $50 \%$ & 4,208 \\
\hline MNH-9:Normal Delivery by a Skilled Attendant +Tetanus Toxoid & $50 \%$ & 6,340 \\
\hline $\begin{aligned} & \text { MNH-18: Tetanus Toxoid +Normal Delivery by a Skilled Attendant } \text { +Active Management + Initial PPH Treatment } \\
&\end{aligned}$ & $50 \%$ & 7,717 \\
\hline MNH-92: Community Newborn Care Package & $80 \%$ & 17,204 \\
\hline MNH-93: Support for Breastfeeding Mothers & $80 \%$ & 14,588 \\
\hline MNH-98:Normal Delivery by a Skilled Attendant & $80 \%$ & 6,734 \\
\hline MNH-100:Normal Delivery by a Skilled Attendant + Tetanus Toxoid & $80 \%$ & 10,145 \\
\hline $\begin{array}{l}\text { MNH-109: Tetanus Toxoid +Normal Delivery by a Skilled Attendant } \\
\text { + Active Management + Initial PPH Treatment }\end{array}$ & $80 \%$ & 12,347 \\
\hline MNH-131: Support for Breastfeeding Mothers + Tetanus Toxoid & $80 \%$ & 22,054 \\
\hline MNH-132: Community Newborn Care Package + ANC & $80 \%$ & 27,784 \\
\hline MNH-183: Community Newborn Care Package & $95 \%$ & 20,429 \\
\hline MNH-184: Support for Breastfeeding Mothers & $95 \%$ & 17,323 \\
\hline MNH-189:Normal Delivery by a Skilled Attendant & $95 \%$ & 7,996 \\
\hline MNH-100:Normal Delivery by a Skilled Attendant + Tetanus Toxoid & $95 \%$ & 12,047 \\
\hline
\end{tabular}

Source: Adam et al., (2005), WHO Choosing Interventions that are Cost Effective. 
According to the above table, the estimated yearly DALYs averted in Butere District whose coverage is $95 \%$ in terms of Community Health Strategy implementation (Intervention), is 1205 .

\subsection{Incremental Cost-effectiveness Ratio of Utilizing CHWs to Promote Maternal Health Services}

-Incremental cost-effectiveness ratio $($ ICER $)=$ Difference in cost/Difference in effectiveness.

- $\quad$ Difference in cost $=$ Cost of promotion of maternal health services in intervention site (Butere District) through CHS - Cost of promotion of maternal in non-intervention (Mumias District) with no CHS.

- Difference in effectiveness= Effectiveness of using CHWs in promoting maternal health services in Butere District (in terms of maternal health indicators) - Effectiveness of not using CHWs in promotion of maternal health services in Mumias District.

\section{$>\mathrm{ICER}(\mathrm{US} \$)=30,124 / 1205=\mathrm{US} \$ 25$ per yearly DALY averted.}

The incremental cost-effectiveness ratio of utilizing Community Health Workers to promote uptake of maternal health services (Focused Antenatal Care and Health Facility delivery) to women of the reproductive age in Butere District was US\$25 per yearly DALY averted. Utilization of Community Health Workers to promote uptake of maternal health services in Butere District was cost effective based on the criteria of "More effective and more costly, with the added benefit worth the added cost."

\subsection{Discounting}

The cost and effects were discounted at a discounting rate of $3 \%$.

\subsection{Sensitivity Analysis}

Sensitivity analysis was done using various allowances paid to the CHWs. A stipend of Kshs. 2,000 as stipulated in the Community Health Strategy Policy and Kshs. 3,000 taking into account the Kenyan annual consumer inflation rate which was $14 \%$ in 2011 . When a motivation of Kshs. 2,000 is paid per CHW, the ICER was US\$41 per yearly DALY averted while when Kshs. 3,000 was paid per CHW, the ICER was US\$50 per yearly DALY averted.

\section{Discussion}

Costs incurred in the utilization of Community Health Workers to promote uptake of maternal health services were in the following 7 major categories:-

1) Human Resource - CHEWs, CHCs, CHWs, Health Facility in charge(s), Administration (Chiefs,
Assistant chiefs, Elders and Community members) and partners (GLUK, MOPHS and $\mathrm{MOH}$ )

2) Allowances- Airtime for mobilization, funds to purchase action materials, community dialogue days and facilitation fees.

3) Time (Indirect cost) - Time spent in relation to Community Health Strategy which would have been spent elsewhere.

4) Stationery- CHEW standard reporting tools, CHWs patient referral books and Household registers.

5) Motivation- Badges, caps, bags, T-shirts, stipend, consideration of $\mathrm{CHWs}$ when hiring subordinate staff in Health Facility.

6) Fixed Asset/ Infrastructure - Office space, computers, motor bikes.

7) Others- Security, Insurance, Trainings and CHWs first AID kits.

Health system costs incurred during utilization of CHWs to promote uptake of maternal health services were: - human resource, allowance and time while the societal costs were: human resource, allowance, time, stationery, motivation, fixed asset/ infrastructure and others, [13].

When costing utilization of CHWs in the implementation of CHS, costs of having community dialogue days, action days, outreaches, household visits and updates were calculated as this were the major component of CHS. The society (community) bore much of the cost (time-Indirect cost) which indicated ownership of the strategy at the grass root level while the health system bore much cost in terms of supportive supervision. The annual cost incurred in utilizing Community Health Workers to promote uptake of maternal health services in Butere District is Kshs.2, 500, 612 (US\$ 30,124).

More women had Focused Antenatal Care 53\% in January, 2011 and increased significantly to $66 \%$ in December, 2011. On the other hand, Health Facility Delivery was lower at $35 \%$ and 48\% respectively in January 2011 and December 2011.Even with the implementation of Community Health Strategy, Health Facility Delivery was still below 50\%.

Community Health Workers were effective in the promotion of uptake of maternal health services as a woman in Butere District (Intervention) is who had Focused Antenatal Care had higher odds of delivering in a health facility as compared to a woman in Mumias District (Control) who had lower odds of to delivering in a health facility. CHW interventions are shown to have increased the uptake and coverage of Sulphadoxine Pyrimethamine (SP). For instance $67.5 \%$ of pregnant women were able to access Intermittent Preventive Treatment for malaria during pregnancy (IPTp) in the second trimester [14] as recommended by $\mathrm{WHO}$ and were also able to adhere to the second dose of SP, compared to $39.9 \%$ in the control.

Likewise in another intervention the coverage of the two recommended doses of SP in pregnancy increased from $41.5 \%$ to $82.9 \%$ [15]. These findings were significant as they demonstrated that $\mathrm{CHWs}$ can be effective at increasing 
coverage and access of SP in their local communities. However, there was an unanticipated effect on ANC attendance reduction in the intervention of [15]. Although this was not witnessed in the study by [14] where ANC attendance for the recommended four visits increased from $34.3 \%$ to $41.5 \%$, the use of CHWs in provision of SP may require to be cautiously approached because of this potential effect. Focused Antenatal Care attendance and Health Facility delivery have a moderate positive correlation in Butere District. In the event of up scaling interventions to improve Focused Antenatal Care attendance, then Health Facility delivery will also improve.

However, there is statistically significant difference between Focused Antenatal Care attendance and Health Facility delivery even after implementation of Community Health Strategy intervention. Different strategies need to be put in place to enable more women to deliver in Health Facilities as it is still below the $50 \%$. Sustained efforts to scale up coverage of health facility delivery at birth from the 44\% Sub-Saharan African countries with high child and adult mortality are therefore crucial to meet the millennium development goals for maternal health [16].

The incremental cost-effectiveness ratio of utilizing Community Health Workers to promote uptake of maternal health services (Focused Antenatal Care and Health Facility delivery) to women of the reproductive age in Butere District was US\$25 per yearly DALY averted. Adam et al., 2005, found the ICER for antenatal care intervention to be \$Int27 per DALY averted. Although these services require considerably more resources, they are effective in reducing maternal morbidity and mortality and, as such, are also highly cost effective, [16].

\section{Conclusions}

Utilization of Community Health Workers to promote uptake of maternal health service is a cost effective strategy in Butere District .Community Health Strategy implementation through utilization of $\mathrm{CHWs}$ to promote maternal health services is economically feasible and viable in Butere District, which is a rural setting.

However, different sustainable strategies to upscale Focused Antenatal Care attendance and Health Facility delivery are to be put in place to supplement Community Health Strategy if the Millennium Development Goal for maternal health (MDG 5) is to be achieved.

\section{Acknowledgements}

Our thanks to the many study authors who gave us additional information regarding study designs and interventions.

\section{REFERENCES}

[1] Drummond, F. (2005).Methods for the Economic evaluation of health care programmes. New York: Oxford university press Inc.

[2] Laxminarayan, R., et al. (2006). Intervention cost-effectiveness: overview of main messages. In Disease Control Priorities in Developing Countries, 2nd Ed., edited by D.T. Jamison, et al. New York: Oxford University Press, 35-86.

[3] Musgrove, P., and J. Fox-Rushby. (2006). Cost-Effectiveness Analysis for Priority Setting. In Disease Control Priorities in Developing Countries, 2nd Ed., edited by D.T. Jamison, et al. New York: Oxford University Press, 271-285.

[4] UNFPA, UNICEF and WHO. (2003). Guidelines on monitoring the availability and use of obstetric services. New York.

[5] Walker, D., Jan, S. (2005). How do we determine whether community health workers are cost-effective? Some core methodological issues. Journal of Community Health:June 2005, Volume 30, Issue 3, pp 221-229.

[6] Bryce, J., Victora, G., Habicht P. (2005) on behalf of the MCE-IMCI Technical Advisors. Programmatic pathways to child survival: results of a multi-country evaluation of Integrated Management of Childhood Illness. Health Policy Plan; 20 (suppl 1); 15-17.

[7] Lewin SA, Dick J, Pond P, Zwarenstein M, Aja G, van Wyk B, Bosch-Capblanch X, Patrick M (2005). Lay health workers in primary and community health care. Cochrane Database Syst $\operatorname{Rev}(1)$ :CD004015.

[8] Cesar JA, Cavaleti MA, Holthausen RS, de Lima LG (2002). [Changes in child health indicators in a municipality with community health workers: The cases of Itapirapua Paulista, Vale do Ribeira, Sao Paulo State, Brazil]. Cad Saude Publica, 18(6):1647-1654.

[9] Wang'ombe K. (2005) Economic evaluation in primary health care: The case of Western Kenya community based health care project. SocSci Med (1984); 18(5): 375-385. Make every mother and child count. Geneva: World Health Organization.

[10] Kenya Demographic Health Survey, 2008-2009.

[11] Dower, C., M. Knox, V. Lindler, and E. O’Neil. 2006. Advancing Community Health Worker Practice and Utilization: The Focus on Financing, San Francisco: University of California Center for the Health Professions, National Fund for Medical Education. http://futurehealth.ucsf.edu/Content/29/2006-12 Advancing _Community_Health_Worker_Practice _and_Utilization_The_Focus_on_Financing.pdf.

[12] Viswanathan, M., J. Kraschnewski, B. Nishikawa, L. C. Morgan, P. Thieda, A. Honeycutt, K. N. Lohr, and D. Jonas. 2009. "Outcomes of Community Health Worker Interventions." Evidence Report/Technology Assessment no. 181.Research Triangle Park, NC: RTI International-University of 40 NorthCarolina Evidence-basedPracticeCenter. http://www.ahrq.gov/downloads/pub/evidence/pdf/comhealt hwork/comhwork.pdf. 
[13] Wanzala, M. et al. (2013) Cost Analysis of Community Health Strategy Implementation: Case Study of Butere Disrtict, Kenya. International Knowledge Sharing Platform. http://www.iiste.org/Journals/index.php/RHSS/issue/view/53 7

[14] Mbonye et al. Intermittent preventive treatment of malaria in pregnancy: the effect of new delivery approaches on access and compliance rates in Uganda. Trop Med Int Health. 2007 Apr; 12(4):519-31.
[15] Msyamboza K, Savage E, Kazembe P, Gies S, Kalanda G, D'Alessandro U, Brabin B. Community-based distribution of sulfadoxine-pyrimethamine for intermittent preventive treatment of malaria during pregnancy improved coverage but reduced antenatal attendance in southern Malawi. Tropical Medicine and International Health 2009; 14 (2): 183-189.

[16] Adam T, Lim S, Mehta S. Cost effectiveness analysis of strategies for maternal and newborn health in developing countries. BMJ 2005; 331: 1107. 